\title{
A Prospective Randomized Trial Comparing Excision and Healing by Secondary Intention versus Rhomboid Excision and Limberg Flap Closure in the Treatment of Primary Sacrococcygeal Pilonidal Sinus
}

\author{
Dina Hany, MD; Mohamed M.E. Ibrahim, MD \\ Department of General Surgery, Ain Shams University, Egypt
}

Background: Surgery is the only treatment for symptomatic saccrococcygeal pilonidal sinus. Various surgical techniques are described in literature; yet, the ideal technique is still controversial because of the high recurrence rate that is associated with the majority of the techniques.

Aim of the work: To evaluate Limberg flap closure for the treatment of primary pilonidal sinus by comparing its operative and postoperative outcome with open excision and healing by secondary intention.

Methods: This is a prospective randomized trial which enrolled eighty patients with primary pilonidal sinus from May 2015 to April 2018. The patients were divided into two groups. Group A included forty patients who underwent excision and the wound was left to heal by secondary intention. Group B included forty patients who underwent rhomboid excision and Limberg flap repair.

Results: The operative time was significantly longer in group B. Duration for complete wound healing and return to work were significantly longer in group A. There was no statistically significant difference between both groups neither in recurrence nor in postoperative complications.

Conclusion: Although recurrence was the same with in healing by secondary intention and Limberg flap, yet rhomboid excision and Limberg flap closure for the management of primary pilonidal sinus is better option in terms of wound healing and early return to work.

Key words: Pilonidal sinus, excision, secondary intention, flap closure.

Disclosure: Authors have no conflict of interest, and the work was not supported or funded by any drug company.

\section{Introduction}

Pilonidal sinus disease is a common clinical problem, especially in healthy young adults. Symptomatic disease involves infection under the skin with the most common site being over the saccrococcgeal region. ${ }^{1.2}$

The pathogenesis of pilonidal disease is still debatable. It may be congenital due to failure of coalescence of the primitive ectoderm ${ }^{3}$ or acquired as in Karydakis's theory which states that the loose hair is the main factor as an invader, which applies some force to be inserted into the skin depending on its vulnerability. ${ }^{2.4}$ Other suggested etiological factors include hirsutism, deep natal cleft, obesity, local trauma, familial predisposition, smoking, sedentary lifestyle ${ }^{5.6}$ and occupations with prolonged sittings as drivers. ${ }^{7.8}$

Pilonidal sinus disease can be asymptomatic or symptomatic ${ }^{9}$ with pain in the sacrococcygal region being the most common clinical symptom. ${ }^{7}$ It can present as an acute inflammation or a chronic sinus ${ }^{9}$ where patients' main complaint is staining of the underwear due to the cyst exudation material. ${ }^{10}$
Asymptomatic patients should not undergo any treatment. Symptomatic patients should be treated surgically. ${ }^{11}$ The surgical treatment of chronic pilonidal disease is generally divided into 2 categories: excision of diseased tissue with primary closure (including various flap techniques) versus excision with a form of healing by secondary intention (including marsupialization). ${ }^{12}$ Ideal treatment is still controversial due to high recurrence rates. ${ }^{10}$

The aim of the present study is to evaluate Limberg flap closure for the treatment of primary pilonidal sinus by comparing its operative and postoperative outcome with open excision and healing by secondary intention.

\section{Patients and methods}

This prospective randomized trial was conducted in Ain Shams University Hospitals from May 2015 till April 2018. The trial included eighty patients with sacrococcygeal pilonidal sinus. Patients were randomly divided into two groups.

The first group (group A) included forty patients who 
underwent excision and the wound was left open to heal by secondary intention. The second group (group B) included forty patients who underwent rhomboid excision and Limberg flap repair.

Inclusion criteria included patients diagnosed to have primary pilonidal sinus disease with clinical presentation including chronic discharging sinus, pain and recurrent abscess formation.

Exclusion criteria included patients with BMI $>35$, recurrent pilonidal sinus, unhealthy donor area, extremely large lesion and ASA IV.

Pre operative preparation: Full medical history was taken from the patients including name, age, occupation, marital status, habits of medical importance and complaints. All patients were examined in the outpatient clinic.

Examination included general examination from head to toe and local examination of the buttock area and sinus pits. Routine preoperative investigations were done to evaluate the patients' fitness for anesthesia. An informed consent was taken.

\section{Surgical technique:}

All patients were operated upon in the prone position under spinal or general anesthesia. All patients received I.V. antibiotic of cephalosporin group half an hour before operation. The trunk was slightly jackknifed at the hips and buttocks were retracted with adhesive tape to allow wide exposure of the operative field. Marking of the site and size of the pilonidal sinus was done (Figures 1). The surgical site was shaved on the operation theater. The skin was prepared with $10 \%$ povidone iodine solution.

In the excision and healing by secondary intention group, excision of the whole sinus and all its tracks till reaching the presacral fascia was done (Figure 2).

Hemostasis was done with closure by a pack with antibiotic ointment.

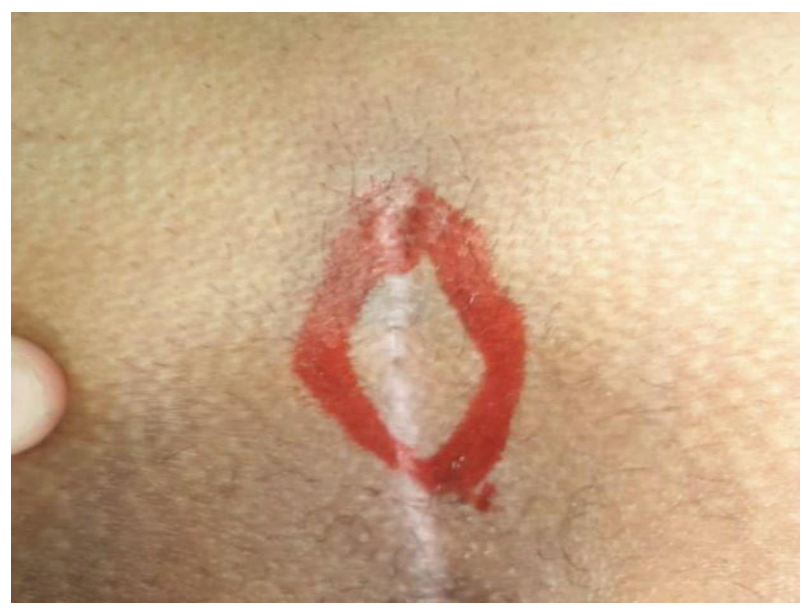

Fig 1. Marking of the sinus.

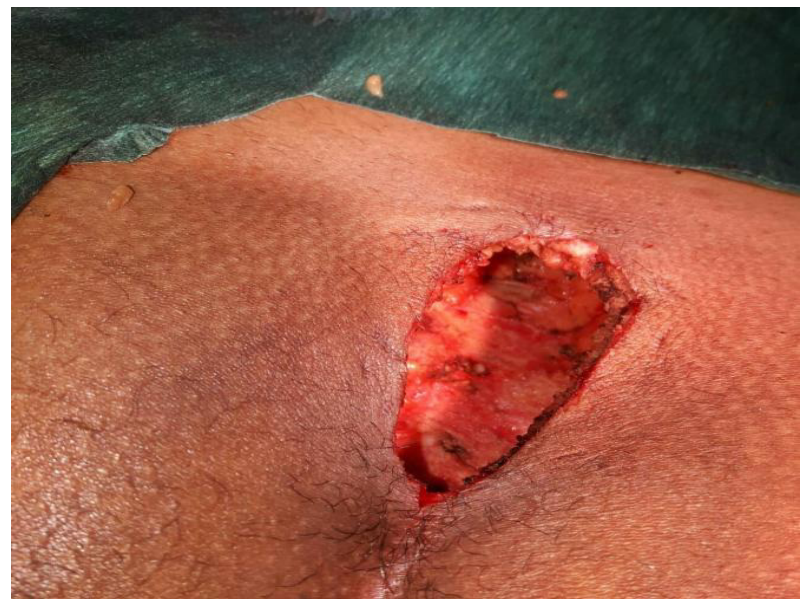

Fig 2: Open excision of the sinus.

In the Limberg flap group, a rhomboid incision with Limberg flap was mapped according to site and size of the pilonidal sinus. A rhomboid incision with limberg flap was done (Figure $\mathbf{3}$ ) followed by excision of the whole sinus and all its tracks (Figures $\mathbf{4 a}, \mathbf{b}$ ) till reaching the presacral fascia. Flap was mobilized and transposed medially so that the defect was closed without tension (Figure 5). A suction drain was placed beneath the flap through separate stab incision, and subcutaneous tissue approximated with polyglycolic acid sutures. The skin was closed separately using 3-0 polypropylene interrupted sutures (Figure 6).

Operative data were recorded immediately after the operation. Operative time was calculated from the time of incision to the completion of the procedure. 


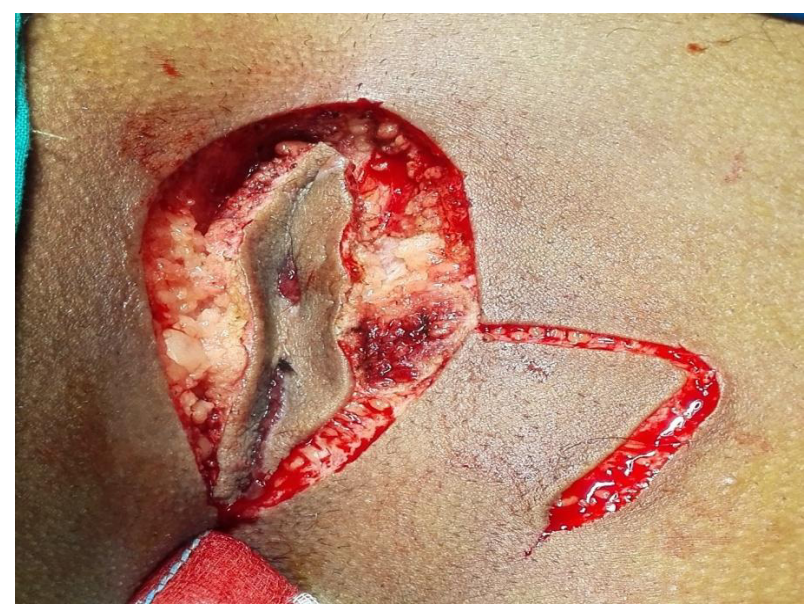

Fig 3: Rhomboid and Limberg flap incision.

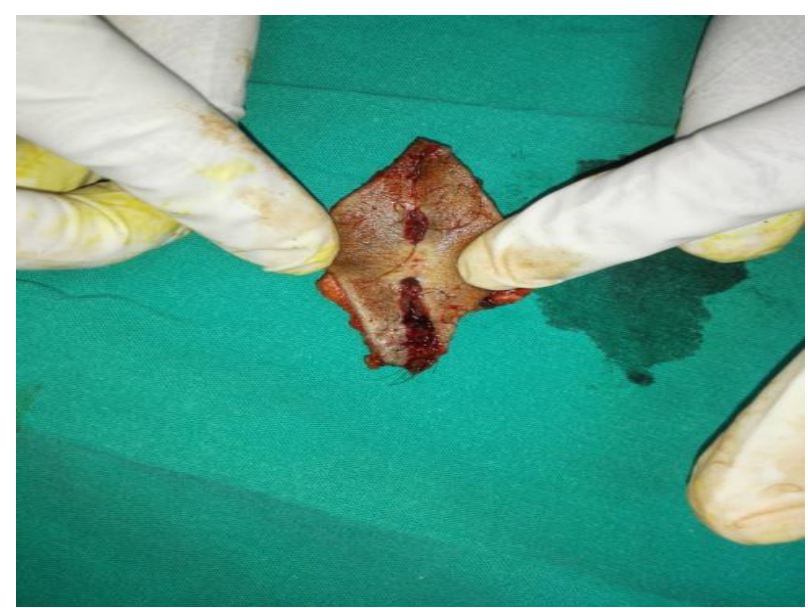

Fig 4a: Skin surface.

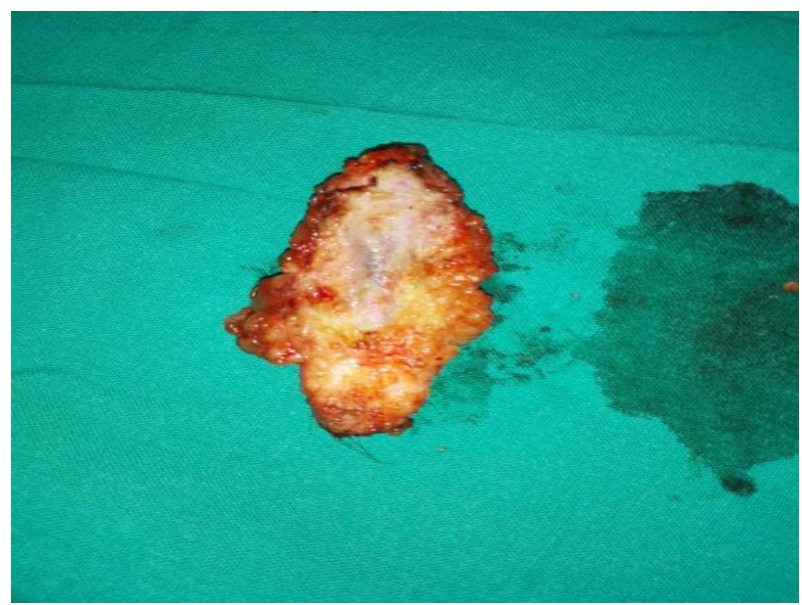

Fig 4b: Deep surface.

Fig 4: Rhomboid shaped specimen with pilonidal pits.

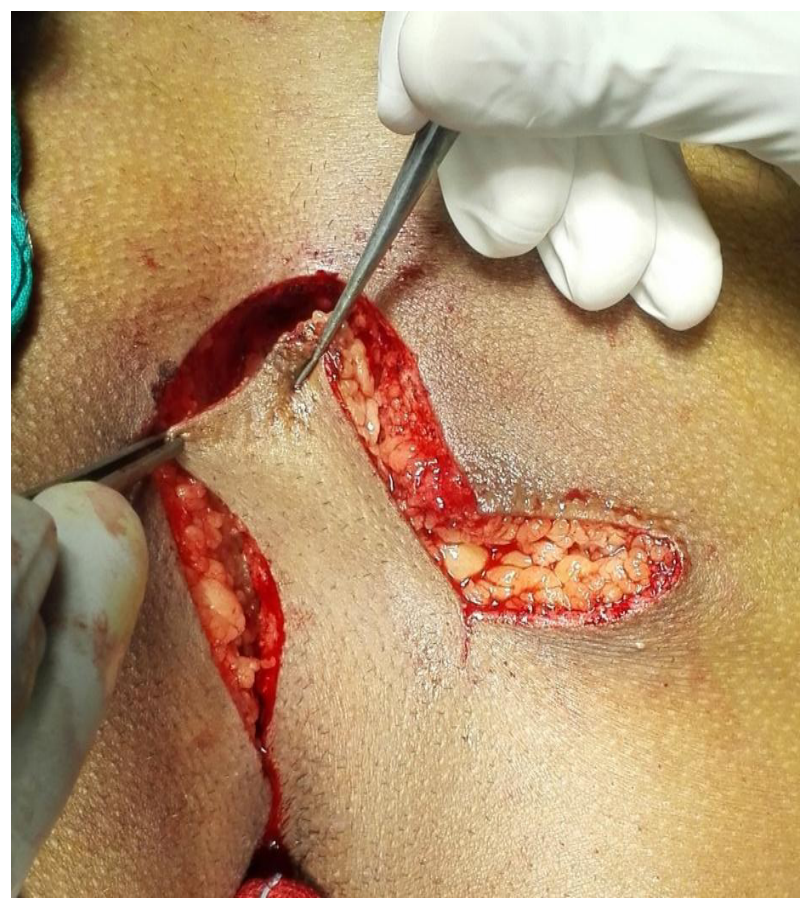

Fig 5: Limberg flap mobilization.

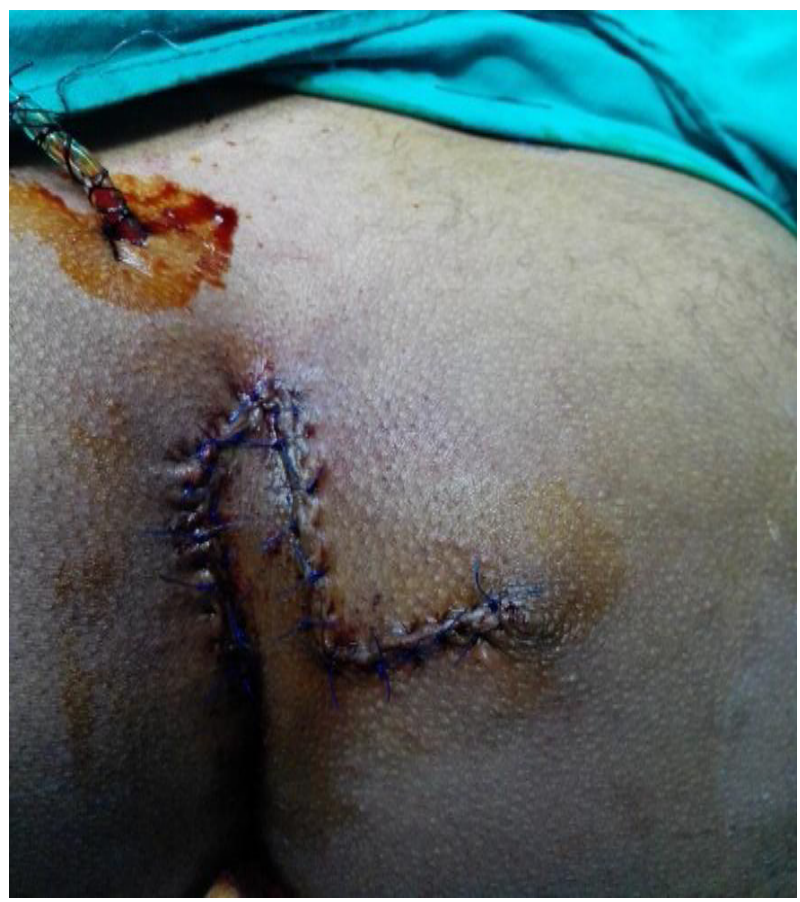

Fig 6: Immediately postoperative.

\section{Post operative follow up}

Patients were discharged on the first postoperative day on oral broad spectrum antibiotic (amoxicillin and clavulanic acid twice daily) and oral analgesic (paracetamol 1 gram every 8 hours) for five days. In group B, alphintern 2 tablets every 8 hours were added for 2 weeks.

For group A patients, dressing was by saline or tap water wash twice daily and application of collagenase ointment and coverage of the wound by a pad.

Follow up at the outpatient clinic was done every 2 
weeks in the 1st month then monthly till wound healing.

For group B patients, dressing was by povidone iodine (betadine) every other day for one week with coverage by sterile gauze and then daily by tincture benzoine with the wound left open for another week. Training for drain pouring was done and the 1st outpatient clinic visit was scheduled for suction drain removal at the $3^{\text {rd }}$ to $5^{\text {th }}$ postoperative day depending upon the amount of drainage. Follow up was then done weekly with half of the stitches removed 2 weeks postoperative and the other half on the $3^{\text {rd }}$ week postoperative in uncomplicated cases. Patients of both groups were instructed to come for follow up 6 month postoperative to detect early recurrence. Postoperative data and complications were recorded during follow up.

Wound infection was defined as purulent discharge from the incision line accompanied by microbiological growth with wound swab culture. Wound dehiscence was defined as gapping of wound edges with neither purulent discharge nor microbiological growth.

\section{Statistical analysis:}

Statistical analysis was performed using IBM SPSS Statistics version 20.0 for Windows. Results were compared by Student $t$ test or Mann- Whitney $U$ test for continuous variables, and chi-square or Fisher exact tests were used for categorical variables. A $p$ value $<0.05$ was considered to represent statistical significance. A $p$ value $<0.01$ was considered to represent high statistical significance.

\section{Results}

Demographic and preoperative data:

The demographic and preoperative data are summarized in Table 1. There was no significant difference between both groups as regard demographic and preoperative data.

Table 1: Demographic and preoperative data

\begin{tabular}{lcccc}
\hline Variable & Group A & Group B & P value & Significance \\
\hline Number of patients & 40 & 40 & & \\
Age & $31 \pm 8.712$ & $30.3 \pm 5.81$ & 0.674 & NS \\
Sex (M:F) & $32: 8$ & $30: 10$ & 0.592 & NS \\
ASA I & $18(45 \%)$ & $10(25 \%)$ & & NS \\
ASA II & $10(25 \%)$ & $16(40 \%)$ & 0.148 & NS \\
ASA III & $12(30 \%)$ & $14(35 \%)$ & & NS \\
Smoking & $30(75 \%)$ & $24(60 \%)$ & 0.152 & NS \\
Family history & $32(80 \%)$ & $30(75 \%)$ & 0.592 & NS \\
Prolonged sitting & $30(75 \%)$ & $32(80 \%)$ & 0.592 & 0.29 \\
Duration of complaint & $19.85 \pm 10.89$ & $22.4 \pm 10.59$ & & \\
\hline
\end{tabular}

\section{Operative data}

Both procedures passed uneventful in the operation theater. Operative time was $22.5 \pm 5.458$ minutes in group $A$ and $44.1 \pm 7.081$ in group $B$; the difference was highly significant $(p<0.01)$ (Fig. 7).

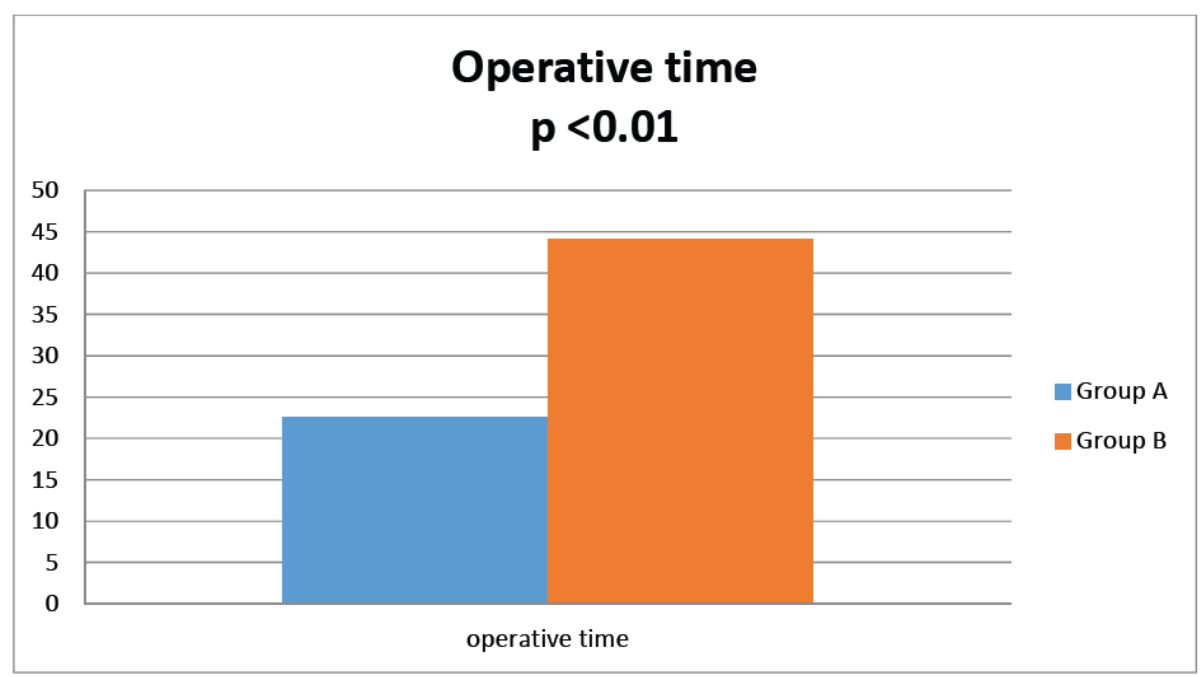

Fig 7: Operative time. 
Postoperative data and follow up

All patients were followed up for 6 months. There were 2 patients with recurrence; $2(5 \%)$ in group $B(p=$ 0.152; not significant). Duration for complete wound healing and return to work were longer in group A

(Figure 8).
Duration for complete wound healing was $40.9 \pm$ 10.446 days in group A and $17.6 \pm 4.465$ in group B. Return to work was $31.15 \pm 9.339$ days in group $A$ and $18.1 \pm 3.828$ days in group $B$.

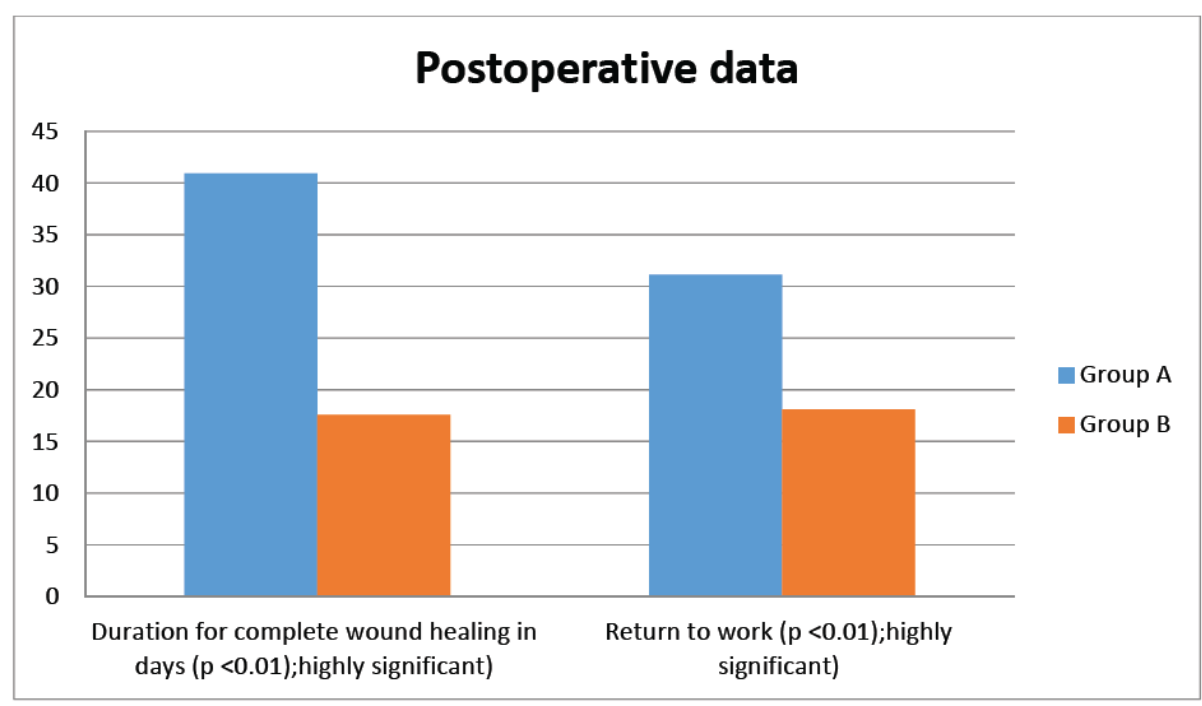

Fig 8: Postoperative data.

\section{Postoperative complications}

Postoperative complications are summarized in
Table 2. All postoperative complications were statistically not significant between both groups.

Table 2: Postoperative complications

\begin{tabular}{lcccc}
\hline Variable & Group A & Group B & P value & Significance \\
\hline Seroma & $0(0 \%)$ & $2(5 \%)$ & 0.152 & NS \\
Wound infection & $0(0 \%)$ & $3(7.5 \%)$ & 0.77 & NS \\
Wound dehiscence & $0(0 \%)$ & $3(7.5 \%)$ & 0.77 & NS \\
\hline
\end{tabular}

\section{Discussion}

Although sacrococcygeal pilonidal sinus is not a lifethreatening condition, yet it adversely affects the quality of life. Although many surgical techniques were described for its management, there is no single best procedure. ${ }^{13}$

The American Society of Colorectal Surgery leaves the method of the treatment to be based on the surgeon and the patient preference, while using the flap-base procedure for the complex and multiple-recurrence chronic disease. ${ }^{12}$

In the present study, healing time and return to normal activity were significantly longer in the excision and healing by secondary intention group

while operative time was significantly longer in the rhomboid excision and Limberg flap group. These results are comparable with other studies in literature. Thus, in a study by Jamal et al14 comparing open excision with secondary healing and Limberg flap, there was significant difference between both groups in terms of operative time and healing time. The operative time was 35 minutes versus 60 minutes and the mean time for complete healing of wound was $120.08 \pm 31.59$ days versus $20.13 \pm 8.99$ days in open excision with secondary healing and Limberg flap groups respectively.

The mean operative time for Limberg flap was variable in different studies; being 36 minutes (range, 22-50 minutes) in Guner et al, ${ }^{15} 34.27 \pm 8$ minutes in Zorlu et al $^{13}$ and 50 minutes (range-30 to 80 minutes) in Singh et al. ${ }^{16}$ 
The 2010 Cochrane systematic review demonstrated significantly longer healing times for open groups (range: 41-91 days) versus primary closure (range, 10-27 days) whether it is done by midline or off midline techniques. ${ }^{17}$

Time to complete healing in open methods takes 1.5 to 3 months. ${ }^{11}$ Daily painful wound care, the slow process of secondary healing and delayed return to work are the main disadvantages of open techniques. ${ }^{11,18}$ The mean healing time in Limberg flap was 11.55 days in Guner et al ${ }^{15}$ study and 11.56 days in Zorlu et $\mathrm{a}^{13}$ study. Earlier completion of postoperative wound healing allows earlier return to normal daily activities. ${ }^{13}$ The mean time to return to work was 19.6 days (range -10 to 30 days) in Singh et al study ${ }^{16}$ on Limberg flap.

In the present study, two patients (10\%) had superficial wound dehiscence. In one patient, the dehiscent wound was diagnosed in the 14th day postoperatively; it was small and healed by daily dressing with collagenase containing ointment.

In the other patient, the dehiscent wound was diagnosed on the $8^{\text {th }}$ day postoperatively; edges were necrotic so they were excised and secondary sutures were done.

The wound dehiscence rate ranged from $0 \%$ Zorlu et $\mathrm{a}^{13}$ study to $23 \%$ McCallum et al ${ }^{19}$ study in different studies on Limberg flap tansposition in pilonidal sinus treatment.

Three patients (7.5\%) had wound infection on their Limberg flap. All presented in the first week postoperatively. Stitches were removed for drainage with antibiotic given according to culture and sensitivity.

Data from randomized trials on overall rates of surgical site infections after flap procedures in management of chronic pilonidal sinus was found low $(0 \%-6 \%)$. $^{12,20-}$ ${ }^{23}$ The overall wound infection rate for Limberg flap ranged from $3 \%$ in Mentes et $\mathrm{al}^{24}$ study to $9.7 \%$ in Guner et al ${ }^{15}$ study.

No patients developed hematoma with Limberg flap in Guner et $\mathrm{al}^{15}$ and Zorlu et al. ${ }^{13}$ studies.

Two patients $(5 \%)$ developed recurrence with the Limberg flap. Overall results of recurrence with the flap procedures in general ranges from $0 \%$ $6 \%{ }^{20-21,23,25-26}$ Recurrence rates following off-midline procedures after follow up period of 12-36 months are reported to range between 0 and $6 \%$ in most publications. . $15,21,27^{2}$

The 2010 Cochrane systematic review demonstrated the open technique had lower recurrence rates; 0.42 range $(0.26-0.66) .{ }^{17}$ Published recurrence rates after open techniques ranges from as low as $2-6 \%$ to $15-35 \%$. This wide range can be explained by patient selection and varying recurrence definitions. ${ }^{11}$

\section{Conclusion}

Although recurrence was the same with in healing by secondary intention and Limberg flap, yet rhomboid excision and Limberg flap closure for the management of primary pilonidal sinus is better option in terms of wound healing, early return to work and overall patient satisfaction.

\section{Limitations}

This study is not without limitations. The small sample size, the limited criteria for patient selection and the short follow up period that can miss late recurrences make this study very limited.

\section{References}

1. Khanna A, Rombeau JL: Pilonidal disease. Clin Colon Rectal Surg. 2011; 24: 46-53.

2. Almajid FM, Alabdrabalnabi AA, Almulhim KA: The risk of recurrence of Pilonidal disease after surgical management. Saudi Med J. 2017; 38 (1): 70-74.

3. De Parades $V$, Bouchard $D$, Janier $M$, Berger $A$ : Pilonidal sinus disease. J Visc Surg. 2013; 150: 237-247.

4. Karydakis GE: Easy and successful treatment of pilonidal sinus after explanation of its causative process. Aust N Z J Surg. 1992; 62: 385-389.

5. Søndenaa K, Anderson E, Soreide JA: Morbidity and short term results in a randomised controlled trial of open compared to closed treatment of chronic pilonidal sinus. Eur J Surg. 1992; 158(6-7): 351355.

6. Doll D, Matevossian E, Wietelmann K, Evers T, Kriner M, Petersen S: Family history of pilonidal sinus predisposes to earlier onset of disease and a 50\% long-term recurrence rate. Dis Colon Rectum. 2009; 52(9): 1610-1615.

7. Humphries $A E$, Duncan JE: Evaluation and management of pilonidal disease, Surg. Clin. $N$. Am. 2010; 90 (1): 113-124.

8. Harlak A, Mentes O, Kilic S, Coskun K, Duman K, Yilmaz F: Sacrococcygeal pilonidal disease: Analysis of previously proposed risk factors. Clinics. 2010; 65 (2): $125-131$.

9. Duman K, Gırgın M, Harlak A: Prevalence of sacrococcygeal pilonidal disease in Turkey. Asian $\boldsymbol{J}$ Surg. 2017; 40(6): 434-437.

10. Sahsamanis G, Samaras, Mitsopoulos G, Deverakis T, Dimitrakopoulos G, Pinialidis D: Semi-closed surgical technique for treatment of pilonidal sinus disease. Annals of Medicine and Surgery. 2017; 15: 47-51.

11. Iesalnieks I, Ommer A, Petersen S, Doll D, Herold 
A: German national guideline on the management of pilonidal disease. Langenbecks Arch Surg. 2016; 401(5): 599-609.

12. Steele SR, Perry WB, Mills S, Buie WD: Standards Practice Task Force of the American Society of Colon and Rectal Surgeons. Practice parameters for the management of pilonidal disease. Dis Colon Rectum. 2013; 56 (9): 1021-1027.

13. Zorlu M, Şahiner IT, Zobac E, Kocak C, Yast AC, Dolapç M: Early results with the Mutaf technique: A novel off midline approach in pilonidal sinus surgery. Annals of Surgical Treatment and Research. 2016; 90(5): 265-271.

14. Jamal A, Shamim M, Hashmi F, Qureshi MI: Open excision with secondary healing versus rhomboid excision with Limberg transposition flap in the management of sacrococcygeal pilonidal disease. J Pak Med Assoc. 2009; 59: 157-160.

15. Guner A, Boz A, Ozkan OF, Ileli O, Kece C, Reis E: Limberg flap versus Bascom cleft lift techniques for sacrococcygeal pilonidal sinus: Prospective, randomized trial. World J Surg. 2013; 37: 20742080.

16. Singh PK, Gohil RK, Saxena N: Limberg flap procedure for sacrococcygeal pilonidal sinus: A prospective study. Int Surg J. 2017; 4(7): 22382242.

17. Al-Khamis A, McCallum I, King BM, Bruce J: Healing by primary versus secondary intention after surgical treatment for Pilonidal Sinus. Cochrane Database Syst Rev. 2010; 1: CD006213.

18. Yoldas T, Karaca C, Unalp O, Uguz A, Caliskan C, Akgun E, Korkut M: Recurrent pilonidal sinus: Lay open or flap closure, does it differ? Int Surg. 2013; 98: 319-323.

19. McCallum IJ, King PM, Bruce J: Healing by primary closure versus open healing after surgery for pilonidal sinus: Systematic review and metaanalysis. BMJ. 2008; 336: 868-871.

20. Abu Galala KH, Salam IM, Abu Samaan KR, et al: Treatment of pilonidal sinus by primary closure with a transposed rhomboid flap compared with deep suturing: A prospective randomized clinical trial. Eur J Surg. 1999; 165: 468-472.

21. Akca T, Colak T, Ustunsoy B, Kanik A, Aydin S: Randomized clinical trial comparing primary closure with the Limberg flap in the treatment of primary sacrococcygeal pilonidal disease. Br J Surg. 2005; 92: 1081-1084.

22. Ertan T, Koc M, Gocmen E, Aslar AK, Keskek M, Kilic $M$ : Does technique alter quality of life after pilonidal sinus surgery? Am J Surg. 2005; 190: 388-392.

23. Muzi MG, Milito G, Cadeddu F, et al: Randomized comparison of Limberg flap versus modified primary closure for the treatment of pilonidal disease. $\mathbf{A m} \boldsymbol{J}$ Surg. 2010; 200: 9-14.

24. Mentes BB, Leventoglu S, Cihan A, Tatlicioglu E, Akin M, Oguz M: Modified Limberg transposition flap for sacrococcygeal pilonidal sinus. Surg Today. 2004; 34: 419-423.

25. Urhan MK, Kücükel F, Topgul K, Ozer I, Sari S. Rhomboid excision and Limberg flap for managing pilonidal sinus: Results of 102 cases. Dis Colon Rectum. 2002; 45: 656-659.

26. Topgül K, Ozdemir E, Kiliç K, Gökbayir H, Ferahköşe Z: Long-term results of Limberg flap procedure for treatment of pilonidal sinus: A report of 200 cases. Dis Colon Rectum. 2003; 46: 1545-1548.

27. Ates M, Dirican A, Sarac M, Aslan A, Colak C: Short and long-term results of the Karydakis flap versus the Limberg flap for treating pilonidal sinus disease: A prospective randomized study. Am J Surg. 2011; 202: 568-573. 